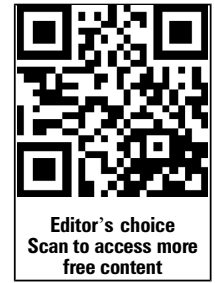

- Additional material is published online only. To view please visit the journal online (http://dx.doi.org/10.1136/ neurintsurg-2013-010656).

${ }^{1}$ Department of Neurology, Emory University School of Medicine, Marcus Stroke and Neuroscience Center, Grady Memorial Hospital, Atlanta, Georgia, USA

${ }^{2}$ Department of Radiology, Emory University School of Medicine, Atlanta, Georgia, USA

\section{Correspondence to} Dr Rishi Gupta, Department of Neurology, Emory University School of Medicine, Marcus Stroke and Neuroscience Center, Grady Memorial Hospital, 49 Jesse Hill Jr Drive SE, Faculty Office Building 393, Atlanta, GA 30303, USA; Rishi.gupta@emory.edu

Received 8 January 2013 Revised 15 February 2013 Accepted 18 February 2013 Published Online First 6 March 2013

\title{
Endovascular Reperfusion and Cooling in Cerebral Acute Ischemia (ReCCLAIM I)
}

\author{
Christopher M Horn, ${ }^{1}$ Chung-Huan J Sun, ${ }^{1}$ Raul G Nogueira, ${ }^{1}$ Vishal N Patel, ${ }^{1}$ \\ Arun Krishnan, ${ }^{2}$ Brenda A Glenn, ${ }^{1}$ Samir R Belagaje, ${ }^{1}$ Tommy T Thomas, ${ }^{1}$ \\ Aaron M Anderson, ${ }^{1}$ Michael R Frankel, ${ }^{1}$ Kiva M Schindler, ${ }_{1}^{1}$ Rishi Gupta ${ }^{1}$
}

\begin{abstract}
Background The efficacy of hypothermia as a neuroprotectant has yet to be demonstrated in acute ischemic stroke. We conducted a phase I pilot study to assess the feasibility and safety of performing intravascular hypothermia after definitive intra-arterial reperfusion therapy (IAT).

Methods ReCCLAIM (Reperfusion and Cooling in Cerebral Acute Ischemia) is a prospective single-arm open-label clinical trial conducted between May and August 2012 at Grady Memorial Hospital. Twenty patients with Alberta Stroke Program Early CT Score (ASPECTS) 5-7 and NIH Stroke Scale (NIHSS) score > 13 were enrolled and treated with intravascular cooling immediately after IAT. The incidence of pneumonia, deep vein thrombosis, cardiac arrhythmias and postoperative hemorrhages was documented for the entire length of stay. Secondary outcomes included blood-brain barrier (BBB) breakdown on gadolinium-enhanced MRIs and 90-day modified Rankin scores (mRS).
\end{abstract}

Results The mean age, median NIHSS score and median final infarct volume were $59.7 \pm 14.6$ years, 19 (IQR16-22) and $78 \mathrm{~cm}^{3}$ (IQR 16-107), respectively. The average time to the target temperature $\left(33^{\circ} \mathrm{C}\right)$ was $64 \pm 50 \mathrm{~min}$. Intracranial hemorrhages were found in three patients, of which one was symptomatic. Evidence of BBB breakdown was observed on 3 of 14 MRIs $(21 \%)$. Six patients died due to withdrawal of care, whereas six patients $(30 \%)$ achieved mRS of $0-2$ at 90 days. In a binary logistical regression model comparing ReCCLAIM patients with 68 historical controls at our institution, hypothermia was protective against intracerebral hemorrhages $(\mathrm{OR} 0.09,95 \% \mathrm{Cl} 0.02$ to $0.56 ; p<0.01)$.

Conclusions Hypothermia can be safely performed after definitive IAT in patients with large pretreatment core infarcts. A phase II study randomizing patients to hypothermia or normothermia is needed to properly assess the efficacy of hypothermia as a neuroprotectant for reperfusion injury.

Trial registration number NCT01585597.

Several clinical trials have failed to show the benefit of neuroprotection in acute ischemic stroke despite promising results in preclinical testing. The failures relate to a multitude of factors, but heterogeneity in stroke subtype and variable reperfusion status are plausible explanations. ${ }^{1}$ The deleterious impact of hyperthermia on outcomes in stroke is well known, ${ }^{2}$ but the clinical efficacy of therapeutic hypothermia has yet to be shown.
The role of mild to moderate hypothermia as a potent neuroprotectant was first proven in patients with anoxic encephalopathy following cardiac arrest. ${ }^{3}$ Preclinical studies in stroke have demonstrated the pleiotropic effects of moderate hypothermia in reducing infarct volume and improving neurological outcomes. ${ }^{4}$ These preliminary data led to clinical trials assessing the feasibility and safety of hypothermia in patients with stroke. ${ }^{5}$ Although these studies showed feasibility in achieving mild to moderate hypothermia, there have been concerns with the rates of pneumonia. Moreover, the patient population selected in these studies was heterogeneous with a mix of patients receiving intravenous tissue plasminogen activator (t-PA), endovascular therapy, as well as those who did not receive definitive reperfusion therapy.

For patients who are poor candidates for intravenous t-PA, intra-arterial therapy (IAT) provides an alternative approach with recanalization rates as high as $89 \% .^{78}$ Reperfusion injury and hemorrhagic conversion, ${ }^{9}$ however, remain a concern with such strategies, in which symptomatic hemorrhage rates have approached nearly $10 \%$ in recent device trials. ${ }^{10} 11$ This highlights the need for effective neuroprotective strategies to prevent the harmful effects of reperfusion.

We therefore initiated a phase I clinical study to address the feasibility of placing patients under mild to moderate hypothermia after definitive endovascular reperfusion. The patients selected for our study were greatly impaired with regard to stroke severity, infarct volume and prognosis, thereby representing a cohort of patients with low anticipated rates of good clinical outcomes. We hypothesized that, if hypothermia could be safely performed in this cohort, then the prospect of using this therapy among a broader population of IAT patients would become ever more promising for future clinical trials.

\section{METHODS}

ReCCLAIM (Reperfusion and Cooling in Cerebral Acute Ischemia) I is a prospective single-arm openlabel clinical trial to assess the feasibility of intravascular hypothermia after definitive reperfusion with mechanical thrombectomy. Patients were enrolled from 10 May 2012 to 28 August 2012. The inclusion criteria were patient age $18-85$ years, Alberta Stroke Program Early CT Score (ASPECTS) 5-7 on pretreatment imaging, ${ }^{12}$ occlusion of the 
M1/M2 middle cerebral artery (MCA) or internal carotid artery (ICA) terminus, presentation $<8 \mathrm{~h}$ from last known normal and treatment with IAT. ASPECTS interpretation was performed by the operator (RG, RN) and adjudicated by an additional study investigator ( $\mathrm{CH}, \mathrm{VP}, \mathrm{TTT})$ prior to enrollment. Patients were enrolled at Grady Memorial Hospital and were required to have a baseline NIH Stroke Scale (NIHSS) score $>13$ with or without intravenous t-PA prior to enrollment. Patients additionally were expected to have a baseline modified Rankin score (mRS) of $0-1$, life expectancy of $>6$ months, successful reperfusion defined as TICI $2 \mathrm{~A}, 2 \mathrm{~B}$ or 3 , along with the absence of a type II parenchymal hematoma $(\mathrm{PH} 2)$ on immediate postreperfusion CT scan of the brain. Exclusion criteria for the study were history of mild cognitive impairment, presence of an inferior venal caval filter, end stage renal failure on hemodialysis, anaphylaxis to iodinated contrast, history of ventricular dysrhythmia leading to cardiac arrest, known hemorrhagic diathesis, platelet count $<5 \times 10^{9} / 1$, international normalized ratio $>1.7$ and/or glucose $<50 \mathrm{mg} / \mathrm{dl}$ or $>400 \mathrm{mg} / \mathrm{dl}$.

\section{Procedure}

Patients were placed under general anesthesia for the procedure and ice packs were placed on the neck, left groin and bilateral axilla to initiate cooling while reperfusion was undertaken. Reperfusion was performed under standard techniques with a Food and Drugs Administration-cleared thrombectomy device (Solitaire FR; Covidien, Mansfield, Massachusetts, USA or Penumbra aspiration catheter; Penumbra Corp, Alameda, California, USA). An Xpert CT of the brain was performed immediately after reperfusion to assess the presence or absence of any intracranial hemorrhage. If the patient was enrolled in the study, a Quattro catheter (Zoll, Chelmsford, Massachusetts, USA) was placed in the inferior vena cava via femoral venous access under sterile conditions. The catheter was placed without ultrasound and sutured into place with a biopatch, and each port was assessed for blood return and ability to flush. The patient was immediately taken to the ICU where the Quattro catheter was connected to the machine for immediate cooling to $33^{\circ} \mathrm{C}$ for $12 \mathrm{~h}$ followed by gradual rewarming at $0.2^{\circ} \mathrm{C} / \mathrm{h}$.

Shivering was prophylactically controlled with the use of buspirone $(30 \mathrm{mg}$ every $8 \mathrm{~h}$ ) given via a nasogastric tube, magnesium sulfate infusion of $12 \mathrm{~g}$ over $24 \mathrm{~h},{ }^{13}{ }^{14}$ and counterwarming techniques consisting of surface warming blankets. Additionally, meperidine boluses of $25 \mathrm{mg}$ and dexmedetomidine continuous infusions $(0.3-0.7 \mu \mathrm{g} / \mathrm{kg} / \mathrm{min})$ were used when the initial measures were unsuccessful in controlling shivering based on the Bedside Shivering Assessment Scale. We recorded specific time points in the continuum of care including intubation, arterial groin access, successful reperfusion, Quattro catheter placement, initiation of hypothermia, time to target temperature, duration of cooling and duration of rewarming.

\section{Imaging}

All patients underwent a follow-up non-contrast CT scan of the brain at $24 \pm 6 \mathrm{~h}$ or earlier if clinically warranted to assess for edema and hemorrhagic changes. Symptomatic hemorrhages were defined according to the ECASS definition as any parenchymal hematoma with space-occupying effects within the infarcted region, together with a clinical deterioration of $\geq 4$ extra points in the NIHSS score compared with the admission baseline within 7 days of treatment. ${ }^{15}$ An MRI of the brain was obtained at 36-72 h after the procedure in patients who were expected to survive using the hyperintense acute reperfusion marker (HARM) protocol that has been described previously. ${ }^{16}$
Gadolinium-dTPA was injected at a dose of $0.1 \mathrm{mmol} / \mathrm{kg}$. The MRI was reviewed for the presence of gadolinium-dTPA and was considered positive if seen in the cerebrospinal fluid spaces of the sulci, ventricles or parenchyma on six or more consecutive slices. Pretreatment ASPECT scores, hemorrhagic transformations and MRI HARM images were reviewed by an independent neuroradiologist (AK) blinded to the angiograms and clinical outcomes.

\section{Definition of pneumonia}

Nosocomial pneumonias were defined according to the guidelines recommended by the American Thoracic Society as any new or developing pulmonary infiltrate or consolidation on radiographic imaging combined with at least two of the subsequent findings (fever $\geq 38^{\circ} \mathrm{C}$, leukopenia/leukocytosis and/or purulent secretions with positive cultures) occurring $48 \mathrm{~h}$ after admission. $^{17}$

\section{Recruitment}

A total of 73 patients were treated with IAT during the recruitment period, 22 of which met the inclusion/exclusion criteria. Consent was obtained for 22 patients, but two were not treated with hypothermia due to the presence of occlusions distal to the M2 segment, thereby meeting an exclusion criterion.

\section{Statistical analysis}

Data pertaining to baseline demographics, neurological severity, pretreatment imaging, hospital length of stay and 90-day mRS were analyzed for all 20 patients. In a secondary analysis, these results were compared with our historical dataset of 68 consecutive patients treated with IAT without hypothermia that would have met the inclusion criteria for ReCCLAIM I but were treated prior to the start of study enrollment. All variables were compared using $\chi^{2}$, Fisher exact, Mann-Whitney U or Student $t$ tests as appropriate. Predictors of hemorrhagic transformation were identified on univariate testing. All variables with $\mathrm{p}<0.20$ were included in our binary logistical regression model, with NIHSS forced into the model to account for initial stroke severity. ${ }^{18}$

\section{RESULTS}

A total of 20 patients with a mean age of $59.7 \pm 14.6$ years and median NIHSS score of 19 (IQR 16-22) were enrolled and analyzed. The distribution of thrombus location was as follows: $8(40 \%)$ carotid terminus, 10 (50\%) M1 MCA, 1 (5\%) M2 MCA, 1 (5\%) tandem occlusion. Average time from symptom onset to groin puncture was $5.4 \pm 1.8 \mathrm{~h}$ with a median baseline ASPECTS of 6 (IQR 6-7). The post-procedure reperfusion grades were TICI 2A: 3 (15\%) patients, TICI 2B: $10(50 \%)$ patients, and TICI 3: 7 (35\%) patients. The median postprocedure infarct volume was $78 \mathrm{~cm}^{3}$ (IQR 16-107). Therapeutic target hypothermia was achieved in $64 \pm 50 \mathrm{~min}$ from the time of cooling initiation (figure 1). The average time spent at the target temperature was $12.1 \pm 2.7 \mathrm{~h}$. In two patients the target temperature was never achieved, one due to an inability to control shivering and one due to a technical issue with the machine.

Of the 20 patients, six (30\%) died due to malignant cerebral edema or the wishes of the family not to pursue aggressive medical care. Three patients received hemicraniectomies, one of which had subsequent withdrawal of care on postoperative day 3 .

Three patients $(15 \%)$ developed new hemorrhages on $24 \mathrm{~h}$ CT scans, of which one $(5 \%)$ was a symptomatic intracranial hemorrhage. A total of 14 patients underwent the HARM 
Figure 1 Time from cooling initiation to target temperature $\left(33^{\circ} \mathrm{C}\right)$ for all 19 and 20 never achieved target temperature. Patient 1 had a body temperature of $33^{\circ} \mathrm{C}$ at onset of initiation from intraprocedural surface cooling measures. ReCCLAIM participants. Patients

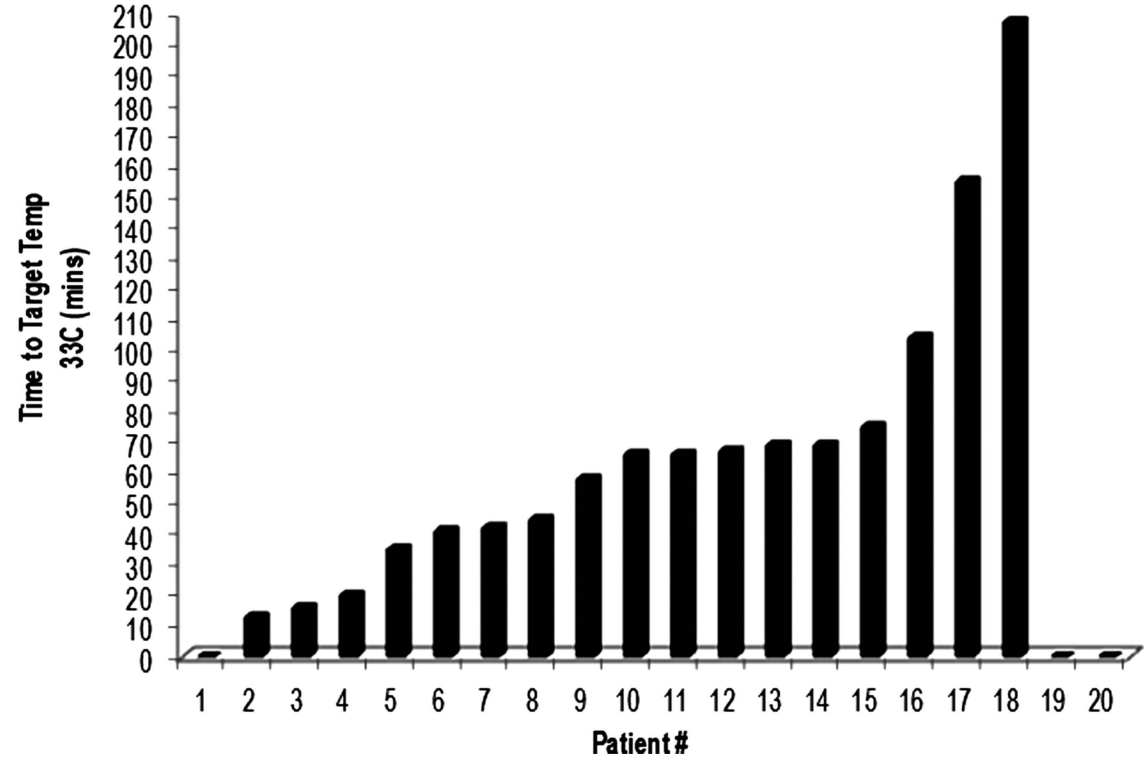

protocol MRI and three (21\%) showed breakdown of the blood-brain barrier (BBB) (figure 2). Five patients could not undergo an MRI due to withdrawal of care, while one patient had a cochlear implant precluding MRI. Of the 11 patients with non-positive HARM results, none had hemorrhagic transformations on $24 \mathrm{~h} \mathrm{CT}$ and five (45\%) had good outcomes at 90 days (mRS 0-2). In contrast, one of the three patients with HARM-positive MRIs had a hemorrhage and one had a good outcome at 90 days.

Medical complications were relatively uncommon in our cohort although pneumonia was present in $25 \%$ of the patients. The other medical complications listed in table 1 appear in line with complications of patients in an ICU setting. Ninety percent of patients had an episode of mild bradycardia and 50\% had occurrences of hypotension, none of which required medical intervention. The median length of ICU stay and time on mechanical ventilation were 4 (IQR 3-6) days and 3 (IQR 2-5) days, respectively.

At the time of discharge, four patients went home (20\%), seven went to acute rehabilitation (35\%), three went to skilled nursing facilities $(15 \%)$ and six patients died from withdrawal of care (30\%). Nearly one-third (30\%) of all patients treated in our study achieved a good outcome at 90 days (mRS 0-2), while $45 \%$ of the patients had acceptable outcomes consistent with mRS 0-3.

We retrospectively reviewed consecutive patients with ASPECTS 5-7 who underwent IAT prior to the start of trial enrollment between October 2010 and May 2012. Of these

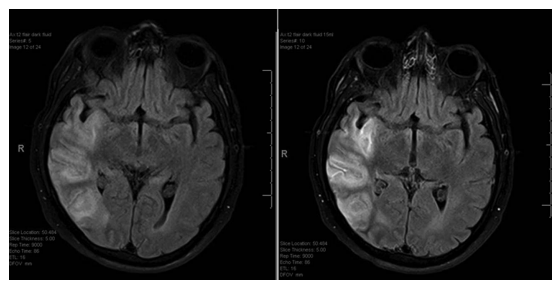

Figure 2 Example of hyperintense acute reperfusion marker positive MRI on T2 Flair imaging with gadolinium enhancement at $24 \mathrm{~h}$ status post-hypothermia. Left panel: pre-gadolinium. Right panel: post-gadolinium. patients, 68 met the inclusion criteria for ReCCLAIM I but were not treated with hypothermia. When compared with these historical controls, ReCCLAIM patients had significantly lower rates of all intracranial hemorrhages $(15 \%$ vs $50 \%, \mathrm{p}<0.01)$. Additionally, differences were noted favoring the ReCCLAIM group with higher rates of placement in acute rehabilitation (35\% vs $22 \%, \mathrm{p}=0.24)$ and better outcomes at 90 days (mRS $0-2: 30 \%$ vs $18 \%, p=0.23$ ), although the differences were not statistically significant. There were no differences between the two groups with respect to demographics, NIHSS scores, time to treatment, infarct volumes, reperfusion success or pneumonia rates (see online supplementary table S1). Stent retriever usage was noted to be greater among the hypothermia patients. In a binary logistical regression model adjusted for age, infarct volume and NIHSS scores, treatment with hypothermia was an independent predictor of protection from hemorrhagic transformation (OR 0.09 , 95\% CI 0.02 to 0.56 ; $<<0.01$, table 2). Hypothermia maintained a trend towards significance in protecting against hemorrhagic transformation when stent retrievers were added to the model (OR $0.167,95 \% 0.02$ to 1.2 ; $\mathrm{p}=0.075)$.

\section{DISCUSSION}

The ReCCLAIM trial is a unique phase I study assessing the feasibility of intravascular cooling following definitive reperfusion therapy with the hope of identifying a salvage strategy to reduce reperfusion injury. The cohort selected represents the most neurologically injured patients prior to reperfusion with extensive core injuries. This preliminary feasibility and safety study showed relatively few medical complications, acceptable hemorrhagic rates and feasibility of this approach for future study. It has provided us with a framework for a phase II study that will randomize patients to normothermia and hypothermia.

Previous studies assessing the potential benefits of hypothermia have focused on a heterogeneous group of stroke patients with or without reperfusion and a broad spectrum of NIHSS scores. ${ }^{5}$ To date, such studies have not shown a signal toward efficacy, resulting in a loss of enthusiasm by the scientific community for hypothermia. The ICTUS $2 / 3$ trial is currently ongoing and is comparing the use of intravenous t-PA plus hypothermia with intravenous t-PA without hypothermia in an 
Table 1 Baseline characteristics of hypothermia patients

\begin{tabular}{|c|c|}
\hline \multicolumn{2}{|l|}{ Demographics } \\
\hline Age, mean (SD) & $59.7(14.6)$ \\
\hline Men, n (\%) & $12(60 \%)$ \\
\hline Hypertension, n (\%) & $16(80 \%)$ \\
\hline Diabetes, n (\%) & $5(25 \%)$ \\
\hline Atrial fibrillation, $\mathrm{n}(\%)$ & $6(30 \%)$ \\
\hline \multicolumn{2}{|l|}{ Neurological severity } \\
\hline Pre-NIHSS, mean (SD) & $19(16-22)$ \\
\hline ASPECTS, median (IQR) & $6(6-7)$ \\
\hline \multicolumn{2}{|l|}{ Treatment } \\
\hline Intravenous tPA given, $\mathrm{n}(\%)$ & $10(50 \%)$ \\
\hline Symptom onset to groin puncture (min), median (IQR) & $309(245-400)$ \\
\hline Symptom onset to cooling initiation (min), median (IQR) & $432(325-534)$ \\
\hline Cooling initiation to hypothermia (min), mean (SD)* & $64(50)$ \\
\hline Duration of cooling (h), mean (SD)* & $12.1(2.7)$ \\
\hline Duration of rewarming $(\mathrm{h})$, mean (SD) & $11.5(4.8)$ \\
\hline \multicolumn{2}{|l|}{ Radiographic imaging } \\
\hline Post-procedural infarct volume $\mathrm{cm}^{3}$, median (IQR) & $78(16-107)$ \\
\hline Asymptomatic hemorrhage (HI1/HI2), n (\%) & $2(10 \%)$ \\
\hline Symptomatic hemorrhage (PH1/2), n (\%) & $1(5 \%)$ \\
\hline HARM status on 36-72 h MRI, n (\%)† & $3(21 \%)$ \\
\hline \multicolumn{2}{|l|}{ Complications } \\
\hline Pneumonia, n (\%) & $5(25 \%)$ \\
\hline Bacteremia, n (\%) & $1(5 \%)$ \\
\hline Deep vein thrombosis, $\mathrm{n}(\%)$ & $1(5 \%)$ \\
\hline Urinary tract infection, $\mathrm{n}(\%)$ & $4(20 \%)$ \\
\hline \multicolumn{2}{|l|}{ Disposition status } \\
\hline NIHSS on discharge, median (SD) $\ddagger$ & $10(3-15)$ \\
\hline mRS on discharge, median (SD) & $4(3-6)$ \\
\hline \multicolumn{2}{|l|}{ 90-day outcomes } \\
\hline Good outcome (mRS 0-2), n (\%) & $6(30 \%)$ \\
\hline Acceptable outcome (mRS $0-3$ ), n (\%) & $9(45 \%)$ \\
\hline
\end{tabular}

anticipated 1600 patients. Hypothermia in patients achieving definitive reperfusion with endovascular approaches, however, has not been the focus of any clinical trial to date.

Our phase I study has provided important advances in the following arenas. We have shown in a patient population with morbidity and mortality that hypothermia can be performed with relative safety. The rates of pneumonia and deep vein thrombosis in our cohort are consistent with previously published percentages in the COOL-AID ${ }^{5}$ and ICTUS-L trials, ${ }^{6}$ despite all our

Table 2 Binary logistic regression model of variables associated with hemorrhagic transformation (HI 1/2 or PH 1/2) after endovascular treatment for acute ischemic stroke

\begin{tabular}{lll}
\hline Variable & OR $(95 \% \mathrm{CI})$ & p Value \\
\hline Hypothermia & $0.09(0.02$ to 0.56$)$ & 0.009 \\
Infarct volume & $1.01(1.00$ to 1.02$)$ & 0.026 \\
Age & $1.02(0.98$ to 1.06$)$ & 0.267 \\
NIHSS & NS & NS \\
\hline
\end{tabular}

Hosmer and Lemeshow test suggests goodness of fit to the model $\left(\chi^{2}=9.9, p>0.05\right)$. $\mathrm{HI}$, hemorrhagic infarct; NIHSS, NIH Stroke Scale; PH, parenchymal hematoma. patients having been intubated for airway protection due to their sizeable pretreatment core infarcts. There were no severe adverse events with regard to ventricular arrhythmias, atrial fibrillation or cardiogenic shock. Although cerebral edema requiring hemicraniectomy was noted in three of our patients within $24-48 \mathrm{~h}$ of successful reperfusion, none of these incidences occurred during the cooling or rewarming phases of the trial.

Successful intravascular cooling was achieved in $90 \%$ of our patients, with only one failing to reach the target temperature due to persistent shivering under propofol sedation. When we changed the primary anti-shivering agent to dexmedetomidine, all subsequent patients achieved the target temperature without complications.

A potential concern with the ReCCLAIM protocol was the prolonged delay between the start of cooling to attainment of the target temperature, which extended to more than $1 \mathrm{~h}$ from the time of Quatro catheter placement. The addition of surface cooling tactics including ice packs and cooling blankets was successful at lowering body temperatures by $0.4^{\circ} \mathrm{C}$ per hour prior to the onset of intravascular cooling, but could not compensate for the overall delay to hypothermia. While the rates of symptomatic hemorrhage are known to be greatest within the first 24-36 h after revascularization, ${ }^{19}$ reperfusion injury probably begins within the first hours of reperfusion. Efforts to achieve hypothermia within this narrow time window may be essential to account for the possibility of BBB disruption immediately after revascularization. Our experiences from ReCCLAIM I reveal that the placement of a cooling catheter prior to endovascular reperfusion leads to minimal delays in achieving definitive recanalization and, thus, has been implemented as part of our protocol for ReCCLAIM II.

Compared with the historical data of all other patients treated with IAT and ASPECTS 5-7 at our facility, patients undergoing hypothermia had significantly lower rates of hemorrhagic transformation and a trend towards higher rates of acute rehabilitation placement and better outcomes at 90 days. These findings are supported by the results of the ASPECTS study group ${ }^{12}$ in which patients with ASPECTS $<7$ had poorer rates of good outcomes (5\%) and higher rates of symptomatic hemorrhage (14\%) than our ReCCLAIM cohort. Additionally, rates of HARMpositive MRIs have previously been shown to be $>40 \%$ in patients with successful reperfusion following ischemic injury ${ }^{16}$ and as high as $50 \%$ in patients treated with IATs. ${ }^{20}$ In our study cohort only 3 out of 14 patients (21\%) had HARM-positive MRIs, suggesting a possible reduction in BBB breakdown. None of the patients screened for ReCCLAIM met the exclusion criteria of an immediate post-reperfusion $\mathrm{PH} 2$ hemorrhage and thus there was no selection bias contributing to the hemorrhage rates documented in the hypothermia cohort. The incidence rates of nosocomial pneumonias following acute strokes were the same in both the ReCCLAIM and historical normothermia groups, highlighting the relative safety of our cooling protocol with respect to respiratory complications.

While these results are encouraging, statements regarding treatment efficacy cannot be definitively concluded given the nature of our phase I trial design. Comparisons with historical data are limited by biases in patient selection, differences in study design, lack of randomization and the presence of potential confounders pertaining to temporal changes in patient care. For example, the use of stent retriever technology became more prevalent at our facility in 2012, resulting in a larger proportion of ReCCLAIM patients treated with these devices compared with the historical controls. The variability in cooling duration among our cohort-due to early withdrawal of care and 
cerebral edema requiring prolonged cooling-may also have biased the observed outcome measures between individual patients. Although such differences in duration did not correlate with rates of hemorrhages in our study, the possibility of a dosedependent effect of hypothermia on $\mathrm{BBB}$ protection remains to be seen.

The external validity of our study may be another limitation given that the results from our ReCCLAIM patients may not be applicable to a patient population with less severe neurological impairment. It is our belief, however, that the rates of medical complications including pneumonia and reperfusion injury are highest among our study cohort due to the severity of the patients' strokes and, thus, the safety profile of a less severely affected stroke population treated with hypothermia would only improve compared with our phase I study.

Finally, five patients in our trial died before a HARM protocol MRI was obtained. It is possible that all five of these patients may have had HARM-positive results following gadolinium injection, thereby increasing the HARM-positive outcome to $40 \%$. While this may dilute the effect of hypothermia on BBB protection from an imaging standpoint, the significance of hypothermia at reducing hemorrhagic transformations remains.

In spite of these limitations, over one-third of our study cohort was accepted into acute rehabilitation centers and showed remarkable recoveries despite extensive pretreatment core infarcts. The combination of HARM-negative MRIs correlated well with the absence of hemorrhage and better neurological outcomes at 90 days. Furthermore, our binary logistic regression model revealed that treatment with hypothermia was independently associated with lower rates of hemorrhagic transformation, regardless of the intraprocedural thrombectomy device used. While hypothermia may not reverse the severity of a patient's core infarct given the delay between cooling and initial clot burden, the benefit of minimizing reperfusion injury presents a promising opportunity to enhance patient care.

In conclusion, we have demonstrated the safety and feasibility of hypothermia in a unique cohort of patients following definitive reperfusion with endovascular approaches. Our preliminary results have set the foundation for pursuing a phase II clinical trial to assess the efficacy of hypothermia as a potential neuroprotectant against reperfusion injury and, ultimately, as a strategy to enhance patient outcomes.

Contributors Writing of manuscript: CMH, RG, C-HJS. Conception of research: CMH, RG, RGN, C-HJS. Statistical analysis: RG, C-HJS. Revision of manuscript: RGN, VNP, AMA, SRB, MRF, TTT. Data collection: CMH, RG, RGN, C-HJS, BAG, KMS. Imaging adjudication: AK.

Competing interests RGN serves on the scientific advisory board for Stryker Neurovascular, Covidien, CoAxia. He also serves on the Data Safety Monitoring Board for Rapid Medical. SRB serves as a consultant for Neural Stem. RG serves on the scientific advisory board for Stryker Neurovascular, Covidien, CoAxia. He is also a member of the Data Safety Monitoring Board for Reverse Medical and Rapid Medical. He is an Associate Editor for the Journal of Neuroimaging. All other authors have no financial disclosures to report.
Ethics approval Ethical approval was obtained from the Emory University Institutional Review Board and consent was obtained from each patient's legal authorized representative prior to enrollment.

Provenance and peer review Not commissioned; externally peer reviewed.

Data sharing statement All enquiries pertaining to access of data from the ReCCLAIM trial may be addressed directly to the corresponding author.

\section{REFERENCES}

1 Savitz SI, Schabitz WR. A critique of SAINT II. Wishful thinking, dashed hopes and the future of neuroprotection for acute stroke. Stroke 2008;39:1389-91.

2 Greer DM, Funk SE, Reaven NL, et al. Impact of fever on outcome in patients with stroke and neurologic injury: a comprehensive meta-analysis. Stroke 2008;39:3029-35.

3 Bernard SA, Gary TW, Buist MD, et al. Treatment of comatose survivors of out-of-hospital cardiac arrest with induced hypothermia. N Engl J Med 2002;346:557-63.

4 Yenari MA, Han HS. Neuroprotective mechanisms of hypothermia in brain ischaemia. Nat Rev Neurosci 2012;13:267-78.

5 De Georgia MA, Krieger DW, Abou-Chebl A, et al. Cooling for acute ischemic brain damage (COOL AID): a feasibility trial of endovascular cooling. Neurology 2004;63:312-17

6 Hemmen TM, Raman R, Guluma KZ, et al. Intravenous thrombolysis plus hypothermia for acute treatment of ischemic stroke (ICTUS-L): final results. Stroke 2010;41:2265-70.

7 Nogueira RG, Lutsep HL, Gupta R, et al. Trevo versus Merci retrievers for thrombectomy revascularisation of large vessel occlusions in acute ischaemic stroke (TREVO 2): a randomised trial. Lancet 2012;380:1231-40.

8 Saver JL, Jahan R, Levy El, et al. Solitaire flow restoration device versus the Merci Retriever in patients with acute ischaemic stroke (SWIFT): a randomised, parallel-group, non-inferiority trial. Lancet 2012;380:1241-9.

9 Stankowski J, Gupta R. Therapeutic targets for neuroprotection in acute ischemic stroke: lost in translation? Antioxid Redox Signal 2011;14:1841-51.

10 Clark W, Lutsep H, Barnwell S, et al. The Penumbra Pivotal Stroke Trial: safety and effectiveness of a new generation of mechanical devices for clot removal in intracranial larger vessel occlusive disease. Stroke 2009;40:2761-8.

11 Smith WS, Sung G, Starkman S, et al. Safety and efficacy of mechanical embolectomy in acute ischemic stroke: results of the MERCI trial. Stroke 2005;36:1432-8

12 Barber PA, Demchuk A, Zhang J, et al. Validity and reliability of a quantitative computed tomography score in predicting outcome of hyperacute stroke before thrombolytic therapy. ASPECTS Study Group. Alberta Stroke Programme Early CT Score. Lancet 2000;355:1670-4.

13 Kizilirmak S, Karakas SE, Akca 0, et al. Magnesium sulfate stops postanesthetic shivering. Ann NY Acad Sci 1997;813:799-806.

14 Zweifler R, Voorhees M, Mahmood A, et al. Magnesium sulfate increases the rate of hypothermia via surface cooling and improves comfort. Stroke 2004;35:2331-4.

15 Hacke W, Kaste M, Fieschi C, et al. Intravenous thrombolysis with recombinant tissue plasminogen activator for acute hemispheric stroke: The European Cooperative Acute Stroke Study (ECASS). JAMA 1995;274:1017-25.

16 Warach $S$, Latour LL. Evidence of reperfusion injury, exacerbated by thrombolytic therapy, in human focal brain ischemia using a novel imaging marker of early blood-brain barrier disruption. Stroke 2004;35(11 Suppl 1):2659-61.

17 American Thoracic Society. Guidelines for the management of adults with hospital-acquired, ventilator-associated, and healthcare-associated pneumonia. Am J Respir Crit Care Med 2005;171:388-416.

18 Paciaroni M, Agnelli G, Corea F, et al. Early hemorrhagic transformation of brain infarction: rate, predictive factors, and influence on clinical outcome. Stroke 2008:39:2249-56.

19 Khatri P, Wechsler L, Broderick J, et al. Intracranial hemorrhage associated with revascularization therapies. Stroke 2007;38:431-40.

20 Kidwell CS, Latour L, Saver JL, et al. Thrombolytic toxicity: blood brain barrier disruption in human ischemic stroke. Cerebrovasc Dis 2008:25:338-43. 